\title{
Study on simplified calculation method for the vibration characters of frame-shear wall structures
}

\author{
Huang Minshui ${ }^{1,2}$ a, Tu Yueya ${ }^{1,2}$ b Zhang Shishun $^{3, c}$ \\ ${ }^{1}$ School of Environment and Civil Engineering, Wuhan Institute of Technology, Wuhan, China \\ ${ }^{2}$ Transportation Research Center, Wuhan Institute of Technology, Wuhan, China \\ ${ }^{3}$ The Hong Kong Polytechnic University, Hong Kong, China) \\ ahuangminshui@tsinghua.org.cn, btuyueya@gmail.com, c10246493@qq.com
}

\begin{abstract}
Keywords: Method of solving the differential equation, method of substructure, vibration characters, frame- shear wall structures, stiffness matrix
\end{abstract}

\begin{abstract}
Two simplified methods are introduced in the paper, in which periods and mode shapes are obtained through solving the story lateral stiffness of frame- shear wall structures, the method of solving the differential equation and the method of substructure. On the basis of assumption for structure, the assembling strategy of mass matrix and stiffness matrix are discussed specially. The periods and mode shapes can be acquired through both methods and the results are compared and analyzed with PKPM and ANSYS. The computation programs are very convenient and can gain the periods and mode shapes quickly. The methods will create some degree of errors, but it is in the scope of acceptance. They are of great reference to structural designers and scientific researchers.
\end{abstract}

\section{Introduction}

With the rapid development of computation theory, the computation methods for high-rise buildings have gained great progress, from the initial vibration analysis of plane frame structure to current space finite element analysis[1], the method of analysis is more and more accurate and complex[2,3]. In actual engineering, there is some degree of difficulty to perform a finite element analysis (FEA) for the whole structure, and FEA is only carried out for parts where there is complex stress distribution. Matrix displacement method is used in the analysis in the high-rise building analysis software, but it is improved. Wall element is introduced in many software, the bar element is still adopted for frame column and beam, but the shear wall is not modeled by thin-wall column, but by shell element which will make the computing results more accurate. The modal reduction is used in the Matrix displacement method for the assembling of lateral stiffness matrix, but modal reduction is complex when the sum of structural elements is large. In the paper, two simplified methods developed based on Fortran are introduced and checked by PKPM and ANSYS, which can be used for solving the dynamic characters, guarantee the validity of computing results and obtain periods and mode shapes quickly and accurately.

\section{Basis of structural dynamics}

Finite element analysis (FEA) is the theoretical basis of the two methods, its basic procedure is: discretization of structure, determination of displacement pattern, analysis of element characteristics and establish and solve equation. Eigen-equation of un-damped free vibration is expressed as[4,5]

$\left|[K]-\omega^{2}[M]\right|\{\delta\}=0$

Where, $[K]$ and $[M]$ is stiffness and mass matrix, $\omega$ is circular frequency; $\{\delta\}$ is eigenvector.

Basic assumptions. The stiffness is rigid in the floor plane and the stiffness is ignored outside the plane. All lateral structures are connected with the rigid floors and the floors can be seen as a horizontal hinged link between the lateral structures. The influence of torsion is not considered and the horizontal displacement of lateral structure at the same floor is equal. 
The plane stiffness of the lateral structure is infinite and its out-of-plane stiffness is very small and can be ignored.

The shear walls can be united into an equivalent shear wall, its synthesized bending stiffness is the total of synthesized bending stiffness of each shear wall. All the frames can be united into an equivalent frame and its synthesized lateral stiffness is the total of synthesized lateral stiffness of each frame. All the coupling beams can be united into a total coupling beam, the restraining moment of the total coupling beam is the sum of restraining moment of each coupling beam.

Method of solving the differential equation. The shear wall and frame are seen as an elastic foundation beam and elastic foundation effectively, the deformation of the shear wall and frame are compatible along with the height.

Setup of differential equation. The relation of the shear wall between the load and internal force, which is a rigid system, is

$$
M_{W}=E I_{W} \frac{d^{2} y}{d x^{2}}
$$

$$
\begin{aligned}
& V_{W}-m=-E I_{W} \frac{d^{3} y}{d x^{3}} \\
& P_{W}+P_{m}=P(x)-P_{F}+\sum \frac{m_{i j}}{h} \frac{d^{2} y}{d x^{2}}=E I_{W} \frac{d^{4} y}{d x^{4}}
\end{aligned}
$$

Where $m_{i j}$ is the restraining moment of the $i$-th coupling Beam of $j$-th floor, $m$ is the total restraining moment of the $j$-th floor, $P_{m}$ is the equivalent of coupling beams.

The relation of the shear wall between the load and internal force is

$$
P_{F}=-\frac{d V_{F}}{d x}=-C_{F} \frac{d y^{2}}{d x^{2}}
$$

Substituting Equation (5) into Equation (4) and make

$$
\lambda=H \sqrt{\frac{C_{F}+\sum \frac{m_{i j}}{h}}{E_{W} I_{W}}} \text { and } \xi=\frac{x}{H}
$$

Then yields

$$
\frac{d^{4} y}{d x^{4}}-\lambda^{2} \frac{d^{2} y}{d x^{2}}=\frac{p(\xi) H^{4}}{E_{W} I_{W}}
$$

Equation (6) is the compatible differential equation between the shear wall and frame.

Solution of stiffness matrix. For the shear frame- shear wall structures, the structure can be simplified as a flexural-shear floor model when solving the periods. The mass is concentrate at the floor and a unit horizontal force $P_{j}=1$ is imposed at every floor, the get the horizontal displacement $\delta_{i j}$ of $i$-th floor, which make up the flexibility matrix [ $\delta$ ]

$$
[\delta]=\left[\begin{array}{l}
\delta_{11} \delta_{12} \cdots \delta_{1 n} \\
\delta_{21} \delta_{21} \cdots \delta_{21} \\
\cdots \cdots \cdots \cdots \\
\delta_{n 1} \delta_{n 2} \cdots \delta_{n n}
\end{array}\right]
$$

The lateral stiffness matrix is defined as the inverse of the flexibility matrix as shown in Eq. 8

$[K]=[\delta]^{-1}$

Solution of differential equation. The floor displacement when horizontal force is imposed can be obtained after differential equation is solved, the solution of Eq. 6 is

$y=C_{1}+C_{2}+A \operatorname{sh} \lambda \xi+B \operatorname{ch} \lambda \xi+y_{1}$ 
Where $y_{1}$ is particular solution and it is different when the load is imposed on the top floor or not.

So, when the load is imposed on the top floor, the solution can be written as

$$
y=\frac{P H^{2}}{E J_{W}}\left[\frac{\operatorname{sh} \lambda}{\lambda^{3} \operatorname{ch} \lambda}(\operatorname{ch} \lambda \xi-1)-\frac{1}{\lambda^{3}} \operatorname{sh} \lambda \xi+\frac{1}{\lambda^{2}} \xi\right]
$$

When the load is not imposed on the top floor, the solution can be written as

$$
y=\frac{P H_{j}^{2}}{E J_{W}}\left[\frac{\operatorname{sh} \lambda_{j}}{\lambda_{j}^{3} \operatorname{ch} \lambda_{j}}\left(\operatorname{ch} \lambda_{j} \xi_{j}-1\right)-\frac{1}{\lambda_{j}^{3}} \operatorname{sh} \lambda_{j} \xi_{j}+\frac{1}{\lambda_{j}^{2}} \xi_{j}\right]
$$

In conclusion, the its basic procedure is: impose unit force $P=1$ at $j$-th floor; determine $\lambda_{j}$ and $H_{j}$; solve the displacement $\delta_{i j}$ with eq. 6 and assemble flexibility matrix $[\delta]$; obtain the lateral matrix through solving the inverse matrix of $[\delta]$.

Error analysis. For the model simplification, the shear wall and frame are linked with coupling beams, so there is some error with the actual stressful condition.

The compatible condition is the displacement of the lateral member at each floor is the same when space cooperating analysis is performed, but when the differential equation is set up, it is assumed that the displacement of the lateral member at any height is the same.

When the concentrated force is not imposed top floor, but on any floor, the influence of the structure above the floor on the structure below the floor is ignored, so there is also certain error.

The actual floor is not ideal rigid slab, so there is some error when the model parameters are solved, such as $I_{W}, C_{F}$ and $m$.

The moment stiffness of shear wall and lateral stiffness of frame is the weighted average of the floor height, so the method is only suitable for the building whose stiffness is uniform along height.

Method of substructure. When horizontal force is imposed and there is no rotation at floor, the lateral structure at the direction can be divided into several substructures, the displacement $y_{j}$ of each substructure at the same floor is equal.

Matrix theory. For substructure $m$, the load vector is expressed as

$\{P\}^{m}=\left(\left[P_{1}^{m} P_{2}^{m} \cdots P_{n}^{m}\right]\right)^{T}$

Because of displacement compatibility, the floor displacement is

$\{y\}^{m}=\left(\left[y_{1}^{m} y_{2}{ }^{m} \cdots y_{n}{ }^{m}\right]\right)^{T}=\left(\left[y_{1} y_{2} \cdots y_{n}\right]\right)^{T}=\{y\}$

The total load vector is

$$
\{P\}=\sum_{m=1}^{r}\{P\}^{m}=\sum_{m=1}^{r}[K]^{m}\{y\}^{m}=\sum_{m=1}^{r}[K]^{m}\{y\}=\left(\sum_{m=1}^{r}[K]^{m}\right)\{y\}
$$

The relation of load and displacement is

$\{P\}=[K]\{y\}$

Comparing Eq. 14 into Eq. 15 yields the total stiffness matrix as

$$
[K]=\sum_{m=1}^{r}[K]^{m}
$$

From the Eq. 16, it can be seen that the total stiffness matrix of structure is the sum of each stiffness matrix.

For the frame-shear wall structure, the shear wall and frame can be seen as two substructures (Fig.1). After the superposition of lateral stiffness matrix of shear wall and frame, the total lateral stiffness matrix of frame-shear wall structure can be obtained. 


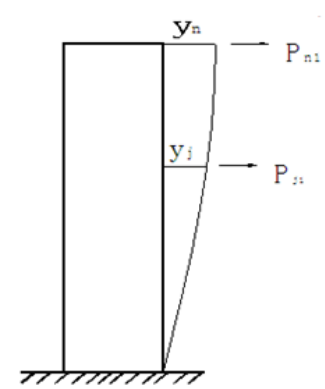

(a) substructure 1 - shear wall

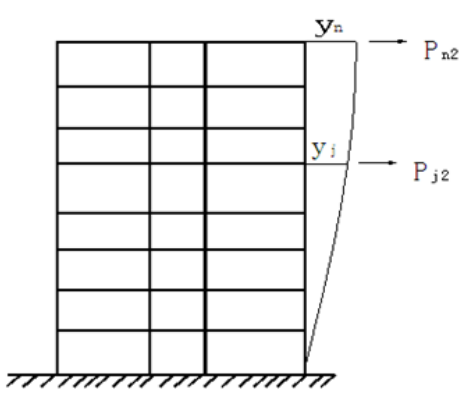

(b) substructure 2 - frame

Fig.1 Two substructures of frame-shear wall

Lateral stiffness matrix of frame. The frame is simplified as layer model. Because the deformation of frame structure is mainly shear, the deformation curve, layer model and $k_{i j}$ are shown as Fig.2.

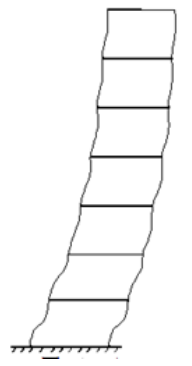

(a) deformation curve

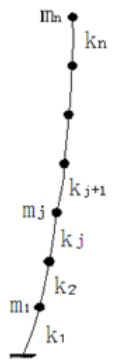

(b) layer model

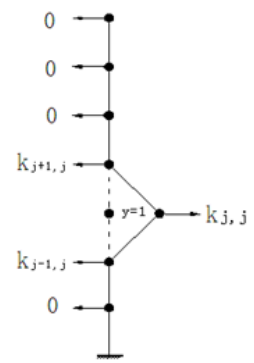

(c) $k_{i j}$

Fig.2 Frame structure

To sum up, the total stiffness matrix for shear model can be expressed as Eq. 17, for the frame structure, $k_{i}$ can be replaced by the value $D$ of every floor.

$$
[K]=\left[\begin{array}{ccccc}
k_{1}+k_{2} & -k_{2} & & & \\
-k_{2} & k_{2}+k_{3} & -k_{3} & & \\
& \ldots & \ldots & \ldots & \\
& & -k_{n-1} & k_{n-1}+k_{n} & -k_{n} \\
& & & -k_{n} & k_{n}
\end{array}\right]
$$

Lateral stiffness matrix of shear wall. The deformation of shear wall structure is mainly flexural, the deformation curve, layer model and $k_{i j}$ are shown as Fig.3.

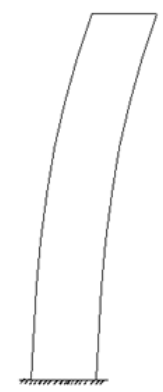

(a) deformation curve

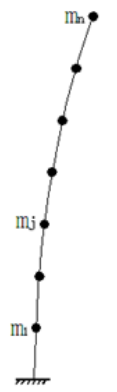

(b) layer model

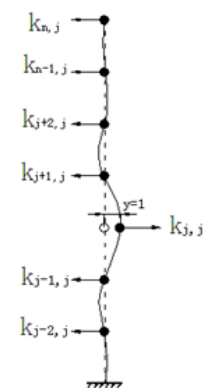

(c) $k_{i j}$

Fig.2 Shear wall structure

For shear wall, the stiffness matrix can be derived with method of energy, after $E I_{i}$ and $G A_{i}$ of each floor is determined. A unit force is imposed at $j$-th floor, and the nodal displacement can be derived with the Eq.18 or Eq. 19

$$
\begin{aligned}
& \Delta=\sum \int \frac{\bar{M} M_{p}}{E I} d s+\sum \int \frac{k \bar{Q} Q_{p}}{G A} d s \\
& \Delta=\sum \int \frac{\bar{M} M_{p}}{E I_{e q}} d s
\end{aligned}
$$

Where $I_{e q}$ is the equivalent moment of inertia of shear wall. 
Error analysis. The layer model is a simplified model, especially for high-rise building, the linear stiffness ratio of beam to column is minor and can be seen as strong column and weak beam, the flexural deformation of frame will relatively large. The deformation of shear model is flexural, but there is also some constituent of shear one, so there is some error unavoidably when it is seen as a flexural model. In addition, the floors are not ideal rigid slabs.

\section{Computational example}

Engineering description. The building is a frame-shear wall structure with 17 storey, the height of major structure is $62 \mathrm{~m}$ and the seismic fortification intensity is 7 degree. The column section is $600 \mathrm{~mm} \times 600 \mathrm{~mm}$, the thickness of shear wall is $250 \mathrm{mmm}$, there are three types of beam section, $250 \mathrm{~mm} \times 600 \mathrm{~mm}, 250 \mathrm{~mm} \times 500 \mathrm{~mm}$ and $250 \mathrm{~mm} \times 400 \mathrm{~mm}$. The building structure system is Cast-in-situ, the strength grade of concrete from storey 1 to storey 6 is C50 and it is C45 above storey 6, the plane arrangement chart of the building is shown as Fig.3.

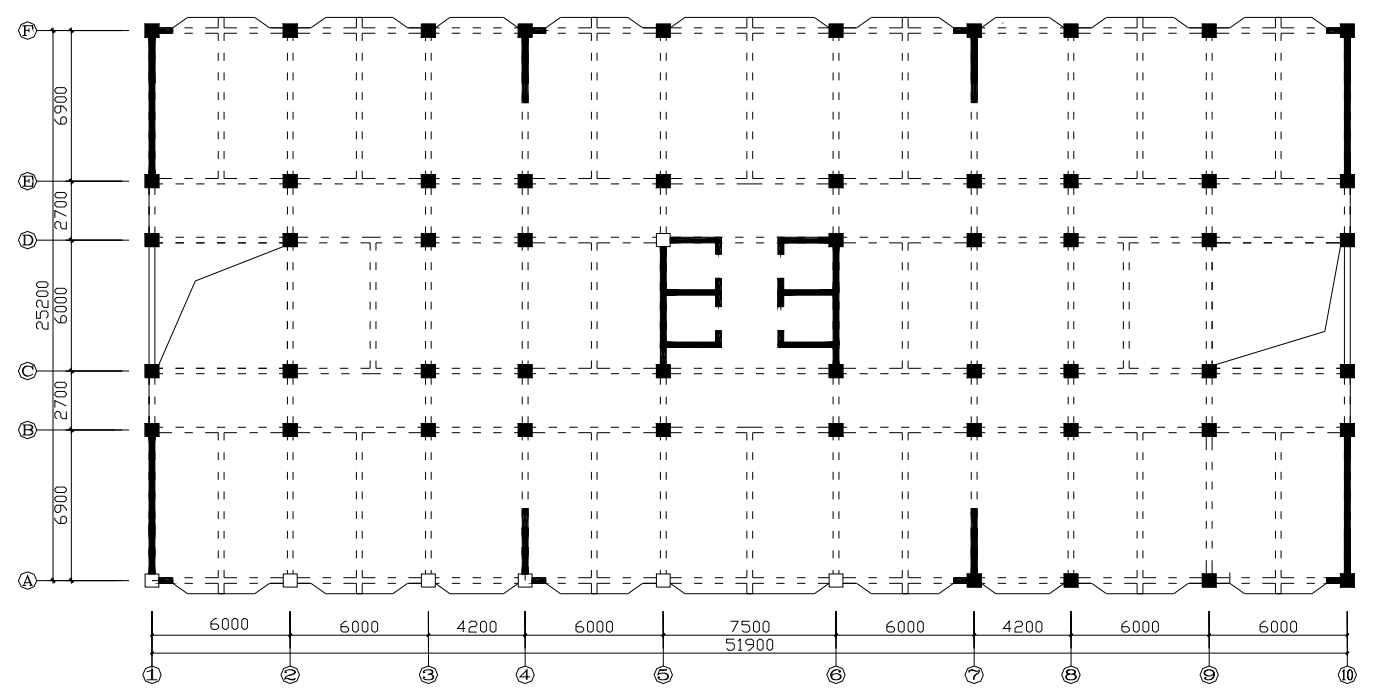

Fig.3 Plane arrangement chart of the building

Analysis results. The first three natural frequencies is shown as Table1, it can be seen that the frequencies calculated with method of solving the differential equation and method of substructure are close to those calculated with commercial software PKPM and ANSYS. The first three mode shapes are shown as Table 2, their shapes are very similar.

Table 1 The first three natural frequencies

\begin{tabular}{|c|c|c|c|}
\hline \multirow{2}{*}{ Method } & \multicolumn{3}{|c|}{ Period (s) } \\
\cline { 2 - 4 } & 1 & 2 & 3 \\
\hline $\begin{array}{c}\text { Method of solving the } \\
\text { differential equation }\end{array}$ & 1.333 & 0.350 & 0.184 \\
\hline $\begin{array}{c}\text { Method of } \\
\text { substructure }\end{array}$ & 1.339 & 0.310 & 0.116 \\
\hline PKPM & 1.346 & 0.340 & 0.153 \\
\hline ANSYS & 1.335 & 0.315 & 0.161 \\
\hline
\end{tabular}

\section{Summary}

An effort has been made to calculate the periods and mode shapes of frame-shear wall structure, which is based on Fortran, the following conclusions can be drawn.

1) From the calculation results, the first natural frequency is very close each other, there are some errors between the second and third frequency, but it is in an acceptable range.

2) From the mode shapes, it can be seen that the mode shape is correct.

3) The two methods is very convenient even though there are some errors, they are of great reference to structural designers and scientific researchers. 
Table 2 The first three natural frequencies

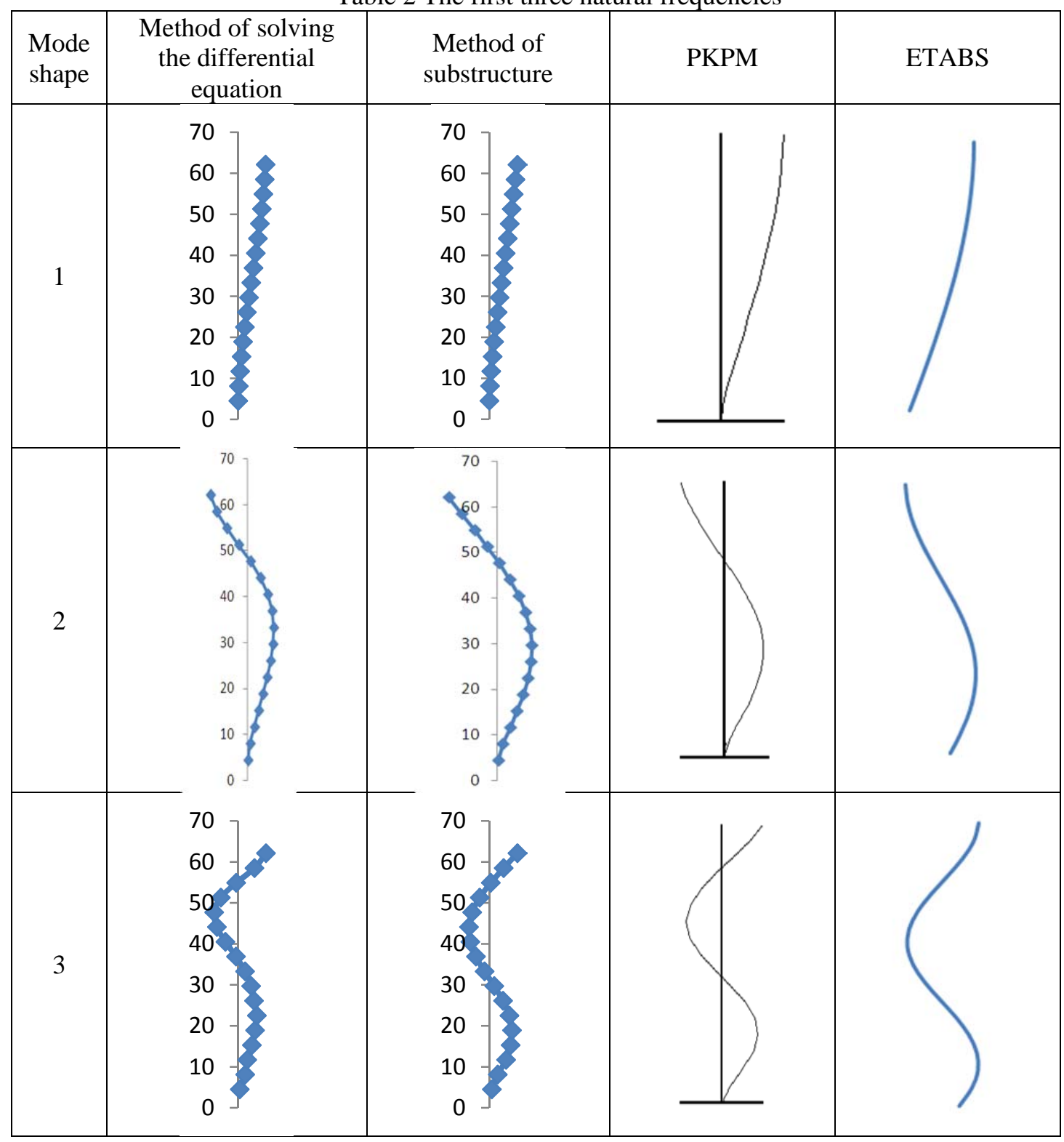

\section{Acknowledgements}

This research was sponsored by Key Science and Technology Project of HBE (D20111512) and Science research foundation of WIT (13115085).

\section{References}

[1] Liu Dahai, Yang Cuiru, Zhong Xigen. Seismic design of high-rise building structures [M]. Beijing: China architecture and building press (1994). (In Chinese)

[2] Bozdogan, Kanat Burak, Ozturk, Duygu. An approximate method for lateral stability analysis of wall-frame buildings including shear deformations of walls[J]. Sadhana - Academy Proceedings in Engineering Sciences, 35(3): p.241-253(2010).

[3] Ye Wenhong, Liang Qizhi. Simplified analysis of frame-shear wall structure considering second order effects[J]. Engineering Mechanics, 16(1): 26-34 (1999). (In Chinese)

[4] Huang minshui, Guo wenzeng, Zhu hongping, Li Lin. Dynamic test and finite element model updating of bridge structures based on ambient vibration [J]. Frontiers of Architecture and Civil Engineering in China, 2(2): 139-144(2008).

[5] Ray W. Clough, Joseph Penzien. Dynamics of structures [M]. New York ; Singapore : McGraw-Hill (1993). 